\title{
AVALIAÇÃO DO PERFIL PEDAGÓGICO DE PROFESSORES NA GRADUAÇÃO POR METODOLOGIAS DE PAÍSES DE ALTO ÍNDICE EDUCACIONAL
}

\author{
Mateus Cardoso Couto ${ }^{1}$; Herman Augusto Lepikson² \\ ${ }^{1}$ SENAI CIMATEC Av. Orlando Gomes, 1845; Salvador/Bahia; 1 mcouto1@gmail.com \\ 2 SENAI CIMATEC Av. Orlando Gomes, 1845; Salvador/Bahia
}

Resumo: O objetivo IV da agenda de 2030 da ONU mostra que é necessária educação equitativa e de qualidade. Apesar de existir essas orientações, o Brasil se encontra num patamar crítico em sua colocação na avaliação educacional do PISA. Esse artigo tem como objetivo demonstrar a evolução docente com a inserção de novos modelos pedagógicos provenientes de países com alto score no PISA. A revisão sistemática foi utilizada como ferramenta norteadora deste estudo para definição do banco de dados por palavras chaves. Concluiu-se que, quando bem adaptadas e com docentes treinados, as metodologias oferecem um impacto positivo para o aumento da qualidade em sala de aula destacando-se como fator positivo para o próximo ranking do Brasil no PISA.

Palavras-Chave: Melhoraria do ensino em sala; Ambientes interativos de aprendizagem; Estratégias de ensino/aprendizagem; Aprendizado colaborativo; Desenvolvimento profissional de professores.

\section{EVALUATION OF TEACHERS' PEDAGOGICAL PROFILE IN UNDERGRADUATION SCHOOLS BY METHODOLOGIES OF COUNTRIES WITH HIGH EDUCACIONAL LEVEL}

\begin{abstract}
Objective IV of the 2030 UN agenda shows that quality and equitable education is needed. Despite these guidelines, Brazil is at a critical level in its placement in the PISA educational assessment. This article aims to demonstrate the teaching evolution with the insertion of new pedagogical models from countries with high score in PISA. The systematic review was used as a guiding tool of this study to define the database by keywords. It was concluded that, when well adapted and with trained teachers, the methodologies offer a positive impact for the increase of quality in the classroom, standing out as a positive factor for Brazil's next ranking in PISA.
\end{abstract}

Keywords: Improving classroom teaching; Interactive learning environments; Teaching/learning strategies; Collaborative learning; Teacher professional development. 


\section{INTRODUÇÃO}

Conforme o objetivo IV da agenda 2030 para o Desenvolvimento Sustentável, documento criado pela Organização das Nações Unidas (ONU), foi aplicada a seguinte meta: "Assegurar a educação inclusiva e equitativa e de qualidade, e promover oportunidades de aprendizagem ao longo da vida para todos" [1].

As discussões globais sobre educação ganharam muita força desde a criação do modelo de avaliação do Programa Internacional de Avaliação de Estudantes (PISA), do inglês Programme for International Student Assessment. No entanto, foi impactante quando o mundo soube que o líder da avaliação do PISA, em dezembro de 2001, foi a Finlândia. Um país que, com cerca de 5.5 milhões de pessoas em sua população total, alcançou o primeiro lugar no maior exame de avaliação global de educação entre outros 31 países membros da Organização para a Cooperação e o Desenvolvimento Econômico (OCDE) [2].

Na mesma edição do PISA de 2001, o Brasil ficou em último lugar. Na edição com dados mais recentes, PISA de 2015, dentre os 70 países participantes, o Brasil obteve as seguintes classificações: $63^{a}$ em matemática, $58^{a}$ em leitura e $65^{a}$ em ciências [2-3].

Ao mesmo tempo onde as discussões educacionais vão se consolidando, o avanço tecnológico atropelou todo firmamento da sociedade pela velocidade e excesso de informação [4]. Um dos maiores impactos é a conectividade de dispositivos e como as informações podem ser acessadas de maneira tão simples. Utilizar os recursos provenientes da quarta revolução industrial em ambientes educacionais pode ser um ótimo catalizador de ensino. Assim sendo, surge o seguinte questionamento: Como é possível adaptar o ecossistema de ensino para melhorar o ranking brasileiro no PISA utilizando a tecnologia como catalizador de nosso progresso?

Dentro deste contexto, o objetivo deste trabalho é realizar uma revisão sistemática sobre o tema visando melhor entendimento sobre a área de estudo.

\section{METODOLOGIA}

Para o desenvolvimento desse estudo foi realizada uma revisão sistemática de acordo com as etapas presentes na Figura 1. Os critérios a seguir foram estabelecidos no desenvolvimento da revisão sistemática: i): foram definidas cinco palavras-chaves em inglês: "Improving classroom teaching"; "Interactive learning environments"; "Teaching/learning strategies"; "Collaborative learning"; "Teacher professional development"; ii) a base de dados foi composta pelos seguintes recursos: Science direct (www.sciencedirect.com), Scielo (www.search.scielo.org) e livros físicos; iii) o intervalo da pesquisa foi definido de 2009 a 2019; iv) foi aferido se a palavra chave estava presente no título dos estudos?; v) foi realizada a interpretação dos resumos e conclusões no caso dos artigos e capítulos no caso dos livros, conforme o filtro estabelecido (Figura 1). 


\section{\begin{tabular}{l|l} 
CIRCULAR ECONOMY & ECONOMIA CIRCULAR
\end{tabular}}

Figura 1. Fluxograma da Revisão sistemática.

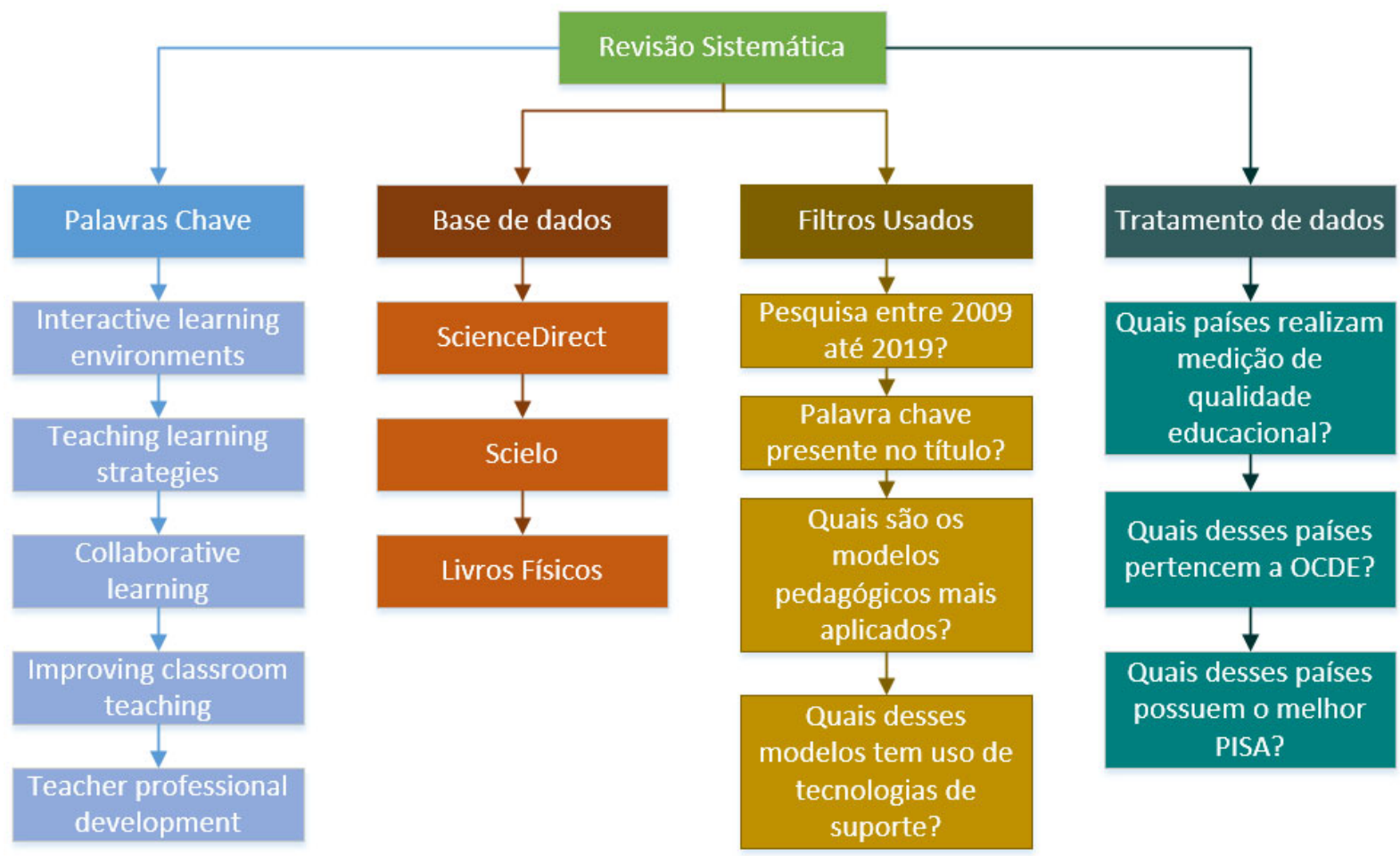

Com a revisão sistemática, a coleta de dados para alavancar os estudos presentes nesse artigo foi feita de maneira simples, organizada e com o foco nos assuntos que realmente importavam.

\section{RESULTADOS E DISCUSSÃO}

Utilizando como base a revisão sistemática foi obtida a etapa seguinte. As pesquisas foram lidas para aplicação da esfera acadêmica, apresentando os processos utilizados. Assim, foi possível obter 35 livros e 76 artigos de uma pesquisa de 358 materiais de estudo que tem alto índice de contribuição para essa pesquisa. $O$ documento da agenda de 2030 da ONU também foi utilizado para definir eixos temáticos de discussão.

A partir da seleção dos artigos encontrados foi construída a Figura 2, que relaciona a quantidade de artigos escolhidos para o desenvolvimento deste estudo de acordo com o seu ano de publicação. A maioria dos estudos são provenientes da Finlândia e Canadá por serem países de alto impacto educacional conforme o mapa presente na Figura 3. 
Figura 2. Relação (quantidades) de artigos e livros por ano de publicação.

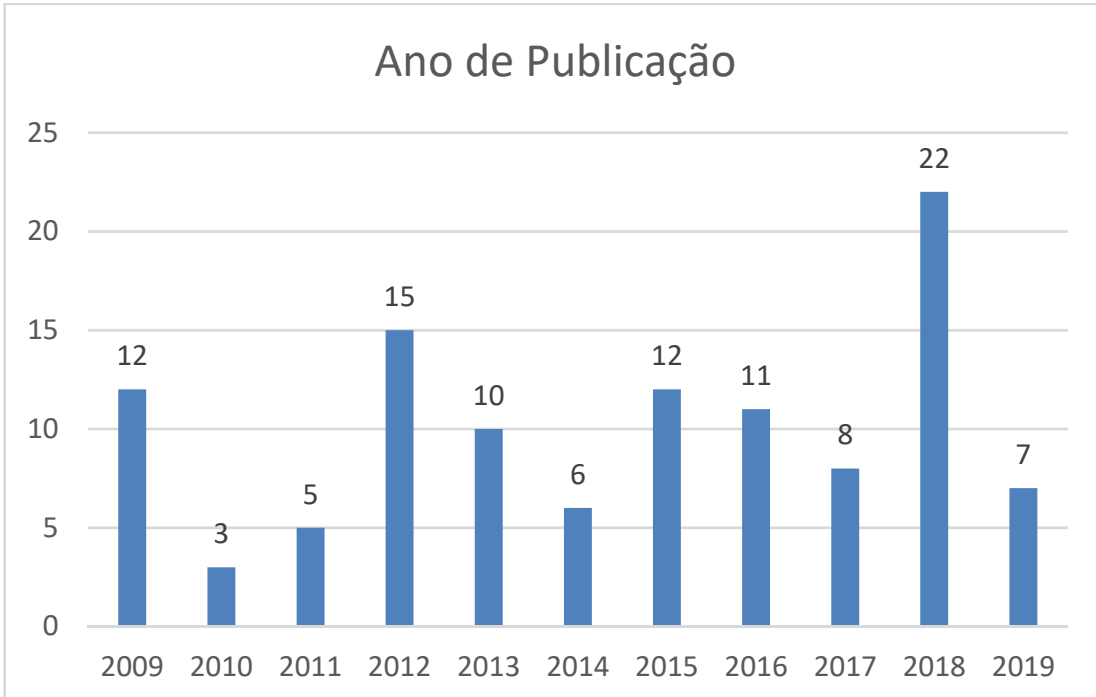

Com as informações da base de dados foi possível identificar que, para atingir algumas submetas do ODS IV, novas metodologias pedagógicas são necessárias, com as seguintes precauções: i) as ferramentas educacionais não podem ser diretamente transplantadas de uma região para outra, elas devem ser adaptadas ao contexto sociocultural do local onde as mesmas serão testadas[5]; ii) é necessário garantir que os docentes possuam casamento de perfil de cargo e perfil da matéria nas atividades exercidas pelos mesmos; iii) cada turma de alunos tem um perfil diferente e isso deve ser respeitado; iv) a graduação recebe alunos que ainda pensam apenas na recompensa quantitativa ao invés de iniciar um desenvolvimento de discernimento crítico, proveniente do modelo de escola conteudista punitiva tradicional brasileira. Foram definidas 4 etapas para implantação desse projeto.

A primeira etapa foi uma coleta de feedback anônimo dos alunos $(n=92)$ referente aos professores selecionados para participar do programa. O feedback consistiu em perguntas sobre o aspecto pessoal e profissional do docente avaliado. Os parâmetros do aspecto pessoal foram compostos por cordialidade, capacidade de autocrítica e postura. Os aspectos profissionais foram compostos por segurança do assunto, domínio da metodologia pedagógica e planejamento. Além disso os estudantes davam opiniões sobre os professores, em geral, via texto aberto no formulário. 
Figura 3. Mapa mundial do ranking PISA 2015.

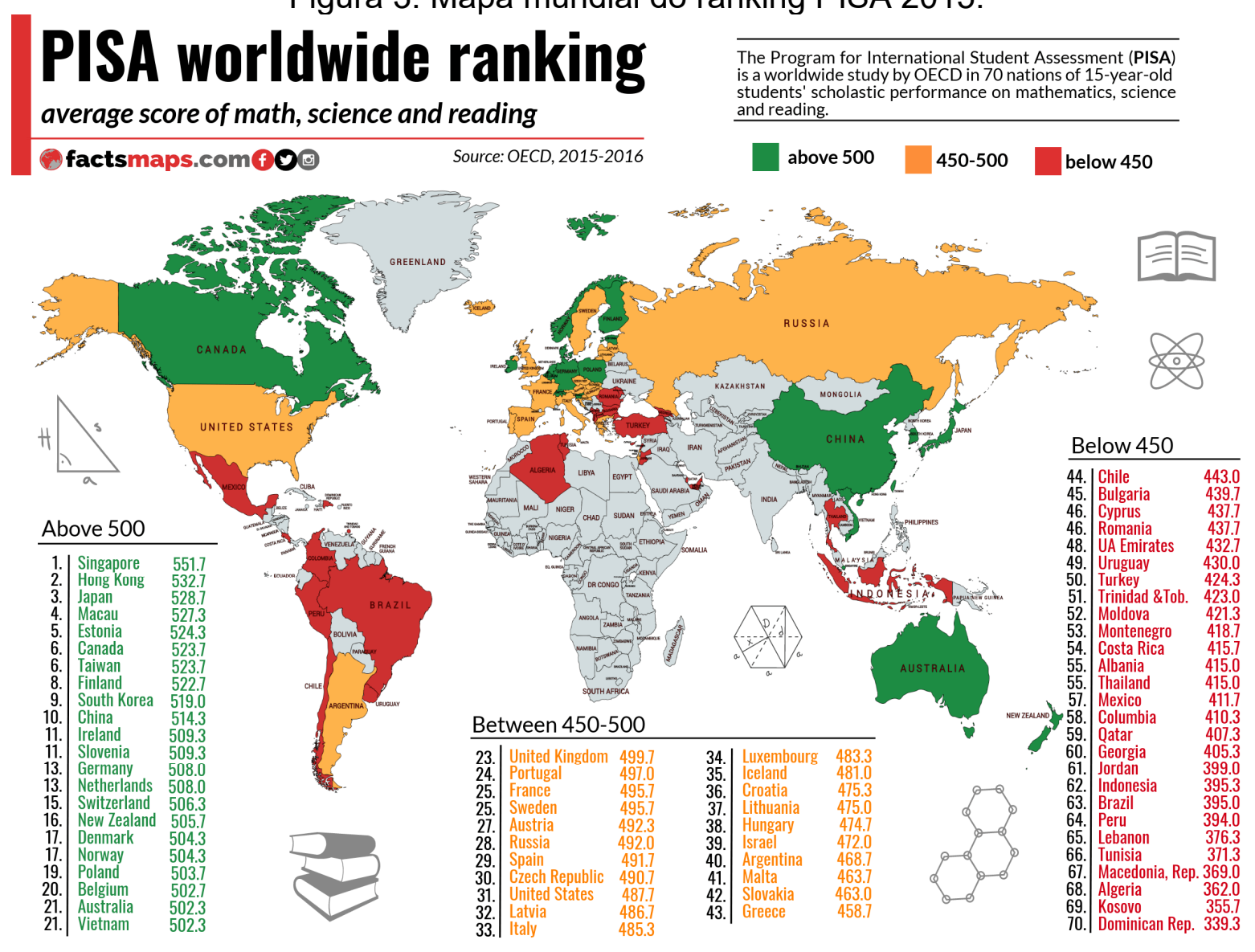

Na segunda etapa ocorreram entrevistas coletivas com o grupo de docentes selecionados para fazer parte do programa $(n=23)$. Na entrevista foi mapeado se o quadro de professores recebeu algum treinamento prévio antes de iniciar a docência. O conjunto de treinamentos que importava para a pesquisa eram sobre metodologias pedagógicas, empoderamento estudantil, princípio da individualidade, planejamento e auto avalição.

A terceira etapa foi a mais delongada e trabalhosa por ter 6 sub etapas. Foi realizado um treinamento individual de cada professor que levou em conta o perfil do mesmo, as características de cada matéria lecionada e as preferências dos alunos de cada turma. Na primeira sub etapa foi construída a roda de competências, conforme Figura 4, de cada docente para a assertividade com as metodologias pedagógicas e os mesmos foram informados sobre os valores educacionais da segunda revolução industrial, que ainda predominam no ecossistema de ensino. Durante a segunda sub etapa foram levantadas todas as dores e problemas referentes aos semestres anteriores para que houvesse uma discussão de caráter reflexivo mediante as falhas cometidas anteriormente. A terceira sub etapa tratou da inserção dos métodos educacionais como PBLs (problem based learning e project based learning), aula invertida, jigsaw, TBL (team based learning), exposição dialogada e dramaturgia pedagógica. A quarta sub etapa se consistiu no empoderamento estudantil, o discente 


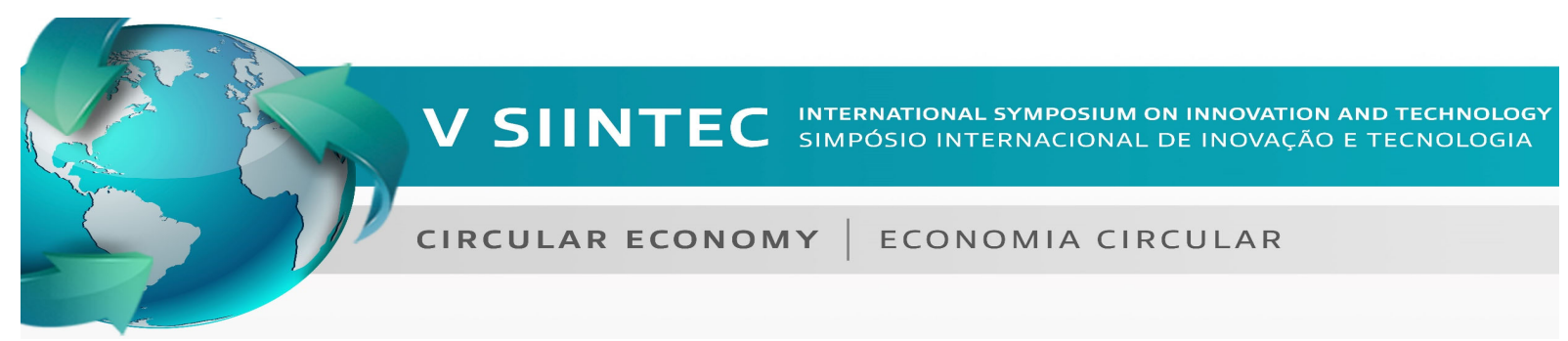

como suporte aos trabalhos mecânicos dos docentes, assim como colocar alunos e professores no mesmo patamar para que hajam feedbacks mútuos. O treinamento por design thinking pela empatia do professor ao se refletir como aluno foi um dos grandes marcos da quarta subdivisão. A quinta sub etapa foi falar sobre as gerações baby boomer, $\mathrm{X}, \mathrm{Y}$ e $\mathrm{Z}$ para que todos entendessem as diferenças das mesmas e como aproveitar o impacto tecnológico a partir da preguiça, um dos maiores marcos por conta do fácil acesso a informação, da geração $Z$. A sexta sub etapa foi um treinamento de Google Suite e Google Classroom para que todos pudessem usar a plataforma a favor de sincronizar melhor os documentos, melhorar a acessibilidade de informações, alinhar cronogramas online, promover um ambiente digital limpo para os alunos e permitir que os mesmos pudessem realizar a leitura com interpretação da curva de aprendizado dos alunos e de si mesmos.

Figura 4. Roda de competências (modelo)

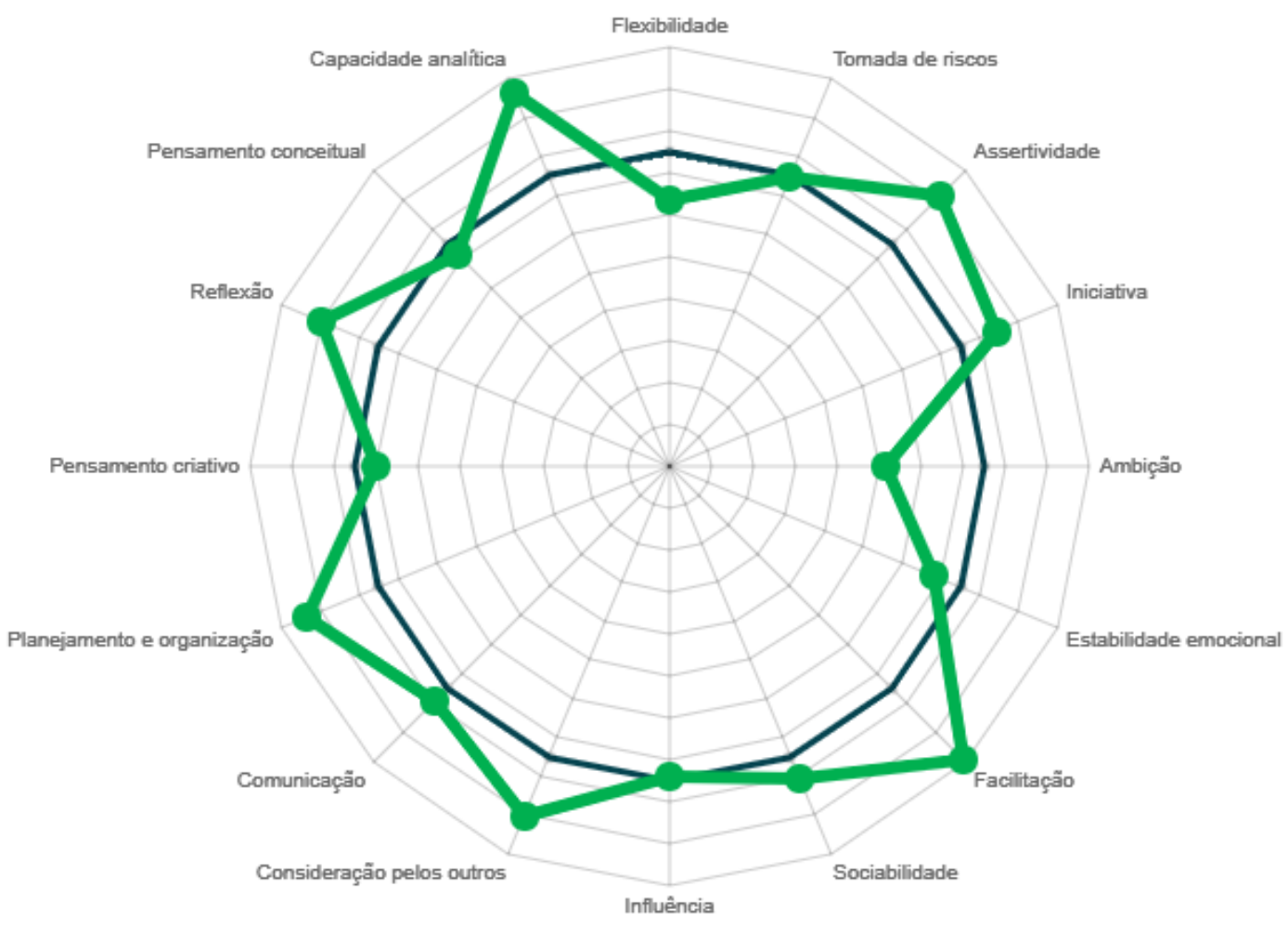

A quarta e a última etapa foi colocar em prática todos os conhecimentos aprendidos previamente pelos professores no semestre seguinte. Os alunos receberam as novas instruções e, em seguida, realizaram um novo feedback com os mesmos parâmetros da primeira etapa. Foi notória uma diferença crucial, tanto na retenção de conteúdo quanto a satisfação em sala de aula (alguns indicadores melhoraram em 40\%). 


\section{CONCLUSÃO}

Os valores educacionais da segunda revolução industrial ainda se encontram presentes nos meios acadêmicos brasileiros. Ao inserir essas etapas dentro dos mecanismos de ensino podemos reduzir a taxa de incidência de uma educação defasada e transformá-la em algo mais moderno com curvas de aprendizado em tempo real dos discentes e docentes.

O papel do professor como fonte de conteúdo foi transformado para um servidor de orientação técnica e de desenvolvimento pessoal no ecossistema acadêmico moderno por conta do impacto tecnológico. Preparar docentes para uma geração que demanda um cuidado especial por conta das influências da conectividade ainda é um grande desafio.

\section{REFERÊNCIAS}

${ }^{1}$ Organização das Nações Unidas. Transformando Nosso Mundo: A Agenda 2030 Para o Desenvolvimento Sustentável. https://nacoesunidas.org/wpcontent/uploads/2015/10/agenda2030-pt-br.pdf. Acesso em: 04 ago. 2019.

${ }^{2}$ Ministério da educação de Portugal. Resultado do Estudo Internacional PISA 2000. https://www.oecd.org/portugal/33685403.pdf. Acesso em: 01 ago. 2019.

3 Instituto Nacional de Pesquisas Espaciais. Brasil no PISA 2015. http://download.inep.gov.br/acoes_internacionais/pisa/resultados/2015/pisa2015_co mpleto_final_baixa.pdf. Acesso em: 01 ago. 2019.

4 ROBINSON, Ken; ARONICA, Lou. Creative Schools: THE GRASSROOTS REVOLUTION THAT'S TRANSFORMING EDUCATION. Londres: Penguin Usa, 2016.

${ }^{5}$ WALKER, Tmothy; SAHLBERG, Pasi. Teach Like Finland. Nova lorque: Norton, 2017. 